\title{
Improving Ergonomics through Haptics
}

\section{Muñoz LM*}

Automatic Control and Computer Engineering Department, Universitat Poletecnica de Catalunya, Spain

Interaction with computers for remote manipulation of objects used in disciplines such as teleoperation, telemedicine or virtual reality, presents problems associated with the absence of proprioceptive and kinesthetic sensations consequently affecting the ergonomics.

In humans, proprioception is the perception of the position and movement of the limbs, perceived through receptors located in muscles, joints, tendons and skin. Proprioceptive information concerning the movement is called kinesthetic. Hence telepropioception is the operator perception to maintain the position of the device and the remote teleoperated environment in relation to his body, i.e. the operator perceives the position of the remote manipulator as if in place. The telekinesthesis is the operator's ability to identify the dynamic movements of teleoperated device, adding speed and feeling of strength to telepropioception. The problems related to telekinesthesis and telepropioception comes from the lose track of the relative speed, direction, position and orientation of the remote manipulator.

Haptesthai in Greek, meaning touch, the tactile sensation is perceived through the skin by thermoreceptors, nociceptors and mechanoreceptors. The latter are those that provide information through touch, pressure, vibration, shape, size, weight, consistency and surface qualities. Each type of stimulus causes a different type of deformation on the skin. Furthermore, the proprioceptive sensation is providing motion information, guidance and strength. The information comes from the somatosensory system, whose receptors are distributed throughout the body, providing a sense of position and movement of body parts together. Receptors found in muscle spindles reporting on muscle length; Golgi receptors on muscle-tendinous junction through passive stretch or active contraction of the fibers provide stretch reflex to maintain posture; Ruffini corpuscles, which provide information on the joint position, direction and speed. The tactile and proprioceptive sensations are closely linked and are difficult to separate. Unlike visual or auditory sensation, haptic stimulation is more difficult to create because their receptors are distributed throughout the body and are very sensitive and small. To create the haptic and proprioceptive or kinesthetic sensations, tactile feedback devices or force feedback are used and an ergonomics methodology can be applied.

There are several technologies used for tactile feedback stimulation over pneumatic, electric and vibrating devices. Some pneumatic devices are made by micro-jets of air, which employ an array of air jets such as those used for representing Braille characters; others are named air rings, based on inflatable devices placed on the fingertip where the pressure on the fingers is controlled and thus simulate tactile presence; and the gloves air capsules, which modify the pressure on tubes or capsules distributed by the fingers and palms, they change the shape stimulating the sense of touch.

There are other devices using vibrating stimulation, electromagnetic devices, based on voice coil (micro- speakers), or motors with eccentric, as used in some game controllers; others based on operation of micropins, either electromagnetic drive, more bulky, piezoelectric type, or through shape memory devices, more compact filament employing alloys as nickel-titanium (nitinol), which shrink when heated. Dome devices use electrostimulation, injecting small electrical pulses through electrodes on the skin, also used to emulate the touch, very compact, although highly invasive.

There are several conventional interface devices used for force feedback, such as mice, joysticks or steering wheels, incorporating internal motors to simulate the effect of obstacle. The latter are used in games or flight simulators and virtual driving.

Another group of devices incorporates gestural input, such as exoskeletons or virtual gloves. In these devices the fingers are positioned with respect the hand by wire, so you can emulate grasping an object with its shape and elasticity. The system can re-form kinesthetic force and simultaneously measure the finger forces to be transmitted to the remote device or to act on the virtual world.

Full kinesthetic feedback exoskeletons arms, also called master arm, typically contain seven degrees of mobility of the human arm. These can be attached on wall or portable on the back.

Other 6 DOF haptic devices arethepen-based masters. They consist of an angular articulated structure, where in its end effector an appendage in the form of pencil is manipulated by the operator.

It has been shown that the use of haptic feedback devices increases the amount of remote sensing, emulating natural kinesthesia and proprioception for handling objects remotely via computer interface. However, the integration of different forms of sensory perception provides the highest degree of approach to Telepresence, which allows perceiving the remote environment like we're really right there.
*Corresponding author: Muñoz LM, Automatic Control and Computer Engineering Department, Universitat Poletecnica de Catalunya, Spain, E-mail: luis.miguel.munoz@upc.edu

Received November 25, 2013; Accepted November 26, 2013; Published December 03, 2013

Citation: Muñoz LM (2013) Improving Ergonomics through Haptics. J Ergonomics 3: e119. doi:10.4172/2165-7556.1000e119

Copyright: @ 2013 Muñoz LM. This is an open-access article distributed under the terms of the Creative Commons Attribution License, which permits unrestricted use, distribution, and reproduction in any medium, provided the original author and source are credited. 